tman (ed.), The Mner Citadel, Oxford, oxford Univ. Press, 1989, pp. 170-189.

27. La metapreferencia de autonomfa es equivalente al segundo interés del más alto ordens de Rawls: el interts por formar, construir y revisar uno mismo una concepción de lo que es bueno para uno mismo.

28. Imaginemos el caso de que si me inyectaran una peculiar droga adictiva me hubiera de despertar cada manana con el intenso deseo de recibir una nueva dosis (el ejemplo es de Derek Parfit, que lo discute con mucha agudeza en Reasons and Persons, Oxford, Clarendon Press, 1984, pp. 497 y $\left.s s_{2}\right)$. Ese deseo no sería inmediatamente doloroso, y alguien le darfa por lo demás siempre rápida satjsfacción. A la larga ssto conllevaría una gran cantidad sumativa de deseos locales satisfechos. Según la apreciación atomista de las preferencias, lo recomendable habria de ser crear artificialmente preferencias fácilmente sacjables (crear un Brave New World). Con todo. si alguien diese ese paso. 10 más probable es que luego yo deseara no haberme convertido en un drogadicto, Pero dado que este deseo no serfa tan perentorio ni pertinaz como mis matutinos anhelos de droga, el total sumativo sería siempre favorable a la iniciativa de convertirme en un drogadicto. Una consideración de las metapreferencias excluye en cambio esta extraña conclusion. Si tanto antes de ser convertido en un drogadicto como después de serlo tengo la metapreferencia de no tener preferencias locales por la droga, por más que estas preferencias fueren satisfechas siempre, la metapreferencia o preferencia global adquiere prioridad sobre esas preferencias locales.

\title{
Los mundos de Nelson Goodman
}

\author{
MANUEL LIZ \\ Universidad de La Laguna
}

La presente nota viene motivada por la reciente publicación en castellano del libro de Nelson Goodman Maneras de hacer mundos (trad, de Carlos Thiebaut), Madrid, Visor, 1990, col. La Balsa de la Medusa.

El titulo original del libro que vamos a comentar es Ways of Worldmaking. Este título queda perfectamente traducido como "Maneras de hacer mundos", y no como wManeras de construcción del mundo", "Formas de construir el mundow o cosas semejantes. Estas altimas traducciones, realizadas en algunos textos, traicionarfan por completo el sentido de la obra de Goodman. Por cierto, el resto de la traducción que del libro realiza Carlos Thiebaut resulta asimismo impecable, conservando perfectamente el tono cstilístico del propio autor.
La primera cdición de este libro de Goodman data de 1978. Doce años después, aún continúa siendo novedad.

En realidad, toda la obra de Nelson Goodman tiene la novedad de los clásicos. Estamos ante uno de los filósofos analíticos que más han marcado el desarrollo de la filosofía analítica contemporánea. Sin embargo, Goodman es un filosofo heterodoxo y fronterizo. Su estilo y la temática de algunos de sus trabajos son difficiles de clasificar dentro de las principales corrientes de la filosofía analítica. Al igual que otros de sus - colegas en Harvard (en otro tiempo, C.I. Lewis, del que el propio Goodman fue discípulo, y, más recientemente, Quine, Putnam, Nozick, Rawls, etc.), más que seguir determinadas corrientes, las inaugura. 
1. "Ways of Worldmaking" en el contexto de otros trabajos de Goodman

Antes de referimos al trabajo de Goodman que nos ocupa, sera conveniente recordar algunas cosas.

En 1951 aparece, basado en su tesis doctoral, su primer libro. Se titulaba The Structure of Appearence. ${ }^{1}$ En el se presenta un sistema fenomenalista de la familia de los sistemas fenomenalistas de Mach, Russell y Camap.2 Estos sistemas exploran la posibilidad de reconstruir lógicamente todo nuestro $\mathrm{co}-$ nocimiento a partir exclusivamente de datos sensoriales. Se trata de un proyecto audaz y radical. Lo sorprendente del sistema de Goodman es su máximo radicalismo dentro de este radicalismo. El sistema de Goodman es fenomenalista, su material básico son los datos sensoriales tal como se le presentan inmediatamente a un sujeto con su peculiar caracter cualitativo. Estos datos sensoriales son llamados qualia (en singular, quale). Un ejemplo de quale sería el aparecer como blanco el papel en el que estoy ahora mismo escribiendo. Su user de color blanco" podría ser, acaso, una propiedad, pero no un quale; su waparecer como de color blancon o, sencillamente, su "parecer blancos sí sería un quale. Esta diferencia es fundamental en todo sistema fenomenalista. Hemos dicho que el sistema de Goodman era fenomenalista, pero también es extensionalista y nominalista. El aparato lógico empleado es cierta versión particular del cálculo de individuos. Su rechazo ontológico a cosas como los significados, las clases, las esencias, los mundos posibles, etc., se convierte desde aquí en un tema constante de toda su obra. El colmo del radicalismo de Goodman surge cuando reconoce explícitamente que no considera al fenomenalismo como una filosofía en sí misma mejor que, por ejemplo, el fisicalismo. El desarrollo de ambos tipos de sistemas no tiene por que ser excluyente. La elección de un sistema sobre otro siempre dependerá de nuestros cambiantes objetivos concretos.

En 1954 publica Fact, Fiction, and Forecast, ${ }^{3}$ tal vez su libro más influyente. En El se enfrenta al problema de la justificación de los condicionales contrafácticos, de la inducción y, en general, de toda generalización (como, por ejemplo, la que acabamos de hacer), de toda generalización descriptiva o normativa. Detengámonos un momento en su especial tratamiento de estas cuestiones. Entre las generalizaciones y los casos particulares debe darse cierto tipo de equilibrio. Los enunciados generales son aceptables mientras no vayan en contra de casos particulares que no estemos dispuestos a ignorar. Por otro lado, los casos particulares son tenidos en cuenta en la medida que no contrarfen las generalizaciones que no estemos dispuestos a abandonar. Mediante este equilibrio reflexivo se justifica toda generalización descriptiva o normativa. Así debe responderse también al viejo escepticismo humeano acerca de la inducción. ${ }^{4}$

El caso es que surge un nuevo problema. El llamado por Goodman nuevo enigma de la inducción. Un equilibrio reflexivo entre generalizaciones y casos particulares permitiria justificar tanto la generalización «todas las esmeraldas son verdes" como, por ejemplo, una generalización del tipo «todas las esmeraldas son verdules", donde el predicado "verdul» (traducimos así el predicado grue de Goodman) se aplicarfa a todo aquello que es azul y a todo aquello que al ser examinado antes de cierto momento $t$ es verde. Respecto de todo momento $t$, nos encontraríamos con multitud de casos particulares en los cuales toda esmeralda 
que sea verde es, asimismo, verdul. Ia totalidad de nuestra evidencia respecto a la generalizacion utodas las esmeraldas son verdes" es, pues, evidencia también respecto a la generalización "todas las esmeraldas son verdules". Una de estas generalizaciones nos parece apropiada y la otra no, pero ¿como rechazar la generalización que nos parece inadecuada? ¿Qué justifica una generalización concreta por encima de todas las alternativas que nuestra imaginación puede llegar a construir?

El problema se complica si consideramos que los predicados "verde» $y$ "azul" pueden ser también redefinidos con ayuda del predicado everdul» $\mathrm{y}$ de otro extraño predicado, del predicado "azurde», donde sazurde» (traducimos así el término bleen de Goodman) se aplica a todo aquello que es verde y a todo aquello que al ser examinado antes de cicrto momento $t$ es azul. Así, "verde" se aplicaría ahora a todo aquello que es azurde y que al ser examinado antes de $t$ es verdul. De manera similar, "azul" se aplicaria a todo aquello que es verdul y que al ser examinado antes de $t$ sea azurde. Tanto "verde» $y$ «azul" como «verdul" $y$ uazurde» podrían, en principio, funcionar como predicados primitivos en nuestros lenguajes $y$ en nuestras organizaciones conceptuales de la experiencia.

Pero, aún hay más. Otra vuelta de tuerca. Si no distinguimos de alguna forma las generalizaciones genuinas de las indeseadas, siempre será posible, a través de ciertos predicados apropiadamente dispuestos, llegar a la conclusion de que cualquier cosa podrá servir como evidencia favorable respecto a cualquier generalización. Veamos. Sea, por ejemplo, aesmerrosa) (traducimos así el término esmerose de Goodman. Curiosamente, volvemos a traducir de un idioma a otro palabras que no existen en ninguno de ellos) un predicado que se aplica a las esmeraldas examinadas antes de cierto tiempo $t$ y a las rosas examinadas después de $t$. La misma evidencia que confirma la generalización de que todas las es. meraldas son verdes confirmara, como vimos, la generalización de que todas las esmeraldas son verdules. Esta misma evidencia, por definición, confirma también la generalización de que todas las esmerrosas son verdules. Pero, también por definición, justamente esa evidencia será evidencia a favor de la hipótesis de que todas las rosas examinadas después de $t$ serán azules. La evidencia a favor de que todas las esmeraldas sean verdes es asimismo evidencia a favor de que todas las rosas serán azules, Sustitúyase en "esmer...» y «ver---» los lugares vacíos por lo que se quiera $y$, de acuerdo a nuestras estipulaciones, obtendremos que aquella evidencia lo es también para la generalización de que todo ... será --..

El problema es importante y sumamente radical. Ya fue señalado también por Hempel $^{5}$ a propósito de los mecanismos de confirmación de nuestras hipótesis. La distinción entre hipótesis confirmadas correcta e incorrectamente, asi como entre generalizaciones genuinas y generalizaciones indeseadas, se resiste a ser trazada mediante criterios meramente formales.

La solucion original que propone Goodman consiste en traer a colación el resto de las generalizaciones ya aceptadas como genuinas. Nuestros predicados "verde" y "azul" han sido mucho más ampliamente utilizados en nuestras gencralizaciones, han sido muchas más veces proyectados (la terminologia es de Goodman) sobre el mundo, que los predicados "verdul" y "azurde". Los primeros predicados, a diferencia de los segundos, se encuen- 
tran muy bien atrincherados (la terminología vuelve a ser de Goodman) en nuestros lenguajes $y$ esquemas conceptuales y están dispuestos a volver a ser fácilmente utilizados, proyectados, siempre que se presente la ocasión. Esta es la única diferencia fundamental que permite considerar ciertas hipótesis o generalizaciones como correctamente confirmadas o genuinas frente a otras que no lo son. La manera peculiar como organizamos nuestra experiencia en ol presente ha de justificarse en relación a nuestra historia lingüística y conceptual. Lo proyectable depende, directamente, de lo que haya sido efectivamente proyectado en el pasado.

Aún debemos recordar algo sumamente importante. Algo que siempre está muy presente en la obra de Goodman. Su interés por la estética y la teoría del arte. La mejor prueba de ello la constituye su libro de 1972 titulado Languages of Art; ${ }^{\circ}$ punto de referencia obligado para buena parte de la estética contemporánea Tanto las formas artísticas como los lenguajes permiten organizar nuestra experiencia y articular nucstro conocimiento del mundo. Aunque el arte se refiera al mundo de una forma típicamente no denotativa, comparte con el lenguaje la capacidad de expresar y ejemplificar nuestros más diversos puntos de vista. Los análisis que Goodman realiza en esta obra acerca de cosas como la metáfora, el estilo o las diversas formas artísticas, en relación a nuestra capacidad de referimos a algo sin denotarlo lingüísticamente, siguen siendo, como decimos, profundamente estimulantes para la actual reflexión estética. Pero, también, para toda aquella reflexión epistemológica que quiera situarse en un continuo entre la ciencia y el arte.

\section{2. "Ways of Worldmaking" en su propia salsa}

En Ways of Worldmaking volvemos a tener gran parte de toda la problematica anterior. ${ }^{7}$ En primer lugar, nos encontramos con un programa filosófico reconstructivo, esta vez mucho más liberal que su anterior sistema fenomenalista aunque siga estando también aquí presente el profundo rechazo de Goodman a cualquier presunta hipóstasis de los significados, las clases, las esencias, los mundos posibles, etc.

Asimismo, nos resultará ya conocida la tenaz insistencia de Goodman en la radical pluralidad de formas como podemos llegar a organizar nuestra experiencia y, asl, llegar a tener mundos alternativos. Lo cual, sin embargo, no conducirá a nìngún relativismo; así como tampoco conducía a un relativismo el fomento de sistemas fenomenalistas y fisicalistas, o la posibilidad de emprender generalizaciones a traves de los predicados "verdul» y "azurde" en lugar de con los predicados averde" $y$ «azul». En el primer caso, cran nuestros objetivos concretos los que, en cada circunstancia, guiaban la elección del tipo de reconstrucción deseable. En el segundo caso, había predicados más proyectables que otros en función de las proyecciones que realmente hubiéramos realizado en el pasado. A fin de desmarcarse del relativismo, el pluralismo de Ways of Worlamaking realiza una pirueta semejante: los mundos que construimos no los construimos de la nada, sino siempre a partir de otros mundos previos.

También nos encontramos en este libro con un persistente interés por acercar los mundos de la ciencia y del arte. Hasta el punto de que, según el mismo Goodman, una de las tesis principales del libro consistirfa justamente en que ael arte no debe tomarse 
menos en serio que las ciencias en tanto forma de descubrimiento, de creación y de ampliación del conocer, en el sentido más amplio de promoción del entendimiento humano, y que, por lo tanto, la filosofía del arte debe concebirse como una parte integral de la metafísica $y$ de la epistemología" (p. 141 de la traducción que estamos comentando).

Hay, pucs, muchos mundos. Algunos de ellos alternativos. $Y$, también, hay muchas formas de construirlos y de llegar a captarlos. Tantas como formas de organizar el conjunto de nuestra experiencia.

En el capítulo 1 de su libro, titulado Palabras, trabajos, mundos, Goodman. discute el problema del pluralismo y señala algunos procedimientos generales de la construcción de mundos; procedimientos como 1) la composición y descomposición a travếs de sus partes, miembros o rasgos, 2) la ponderación de la pertinencia y el énfasis relativo que deben tener ciertos elementos, 3) la ordenación de sus relaciones internas, 4) la supresión y la complementación, o 5) la deformación. Estos procedimientos se ejemplifican más tarde en todo el capítulo 5, titulado «Un rompecabezas para la percepción", ilustrando el viejo tema de la construcción de nuestro mundo perceptivo, y en el apartado 3 del capitulo 6 , en relación a las primeras disputas filosóficas presocráticas acerca de la constitución última de la realidad. Tanto la captación perceptiva de un mundo como su conceptualización teorica no son otra cosa sino formas peculiares de construcción. Goodman, en cualquier caso, no propone ninguna sistematización definitiva de las formas de construir mundos. No puede hacerlo. Las razones que favorecen el pluralismo de mundos favorecen también el pluralismo en todo aquello que pudiera tratar de englobarlos. Como dice Goodman, "no sólo no existe un único mundo sino que tampoco existe un único mundo de mundos" (p. 37 de la traducción que comentamos).

Pero, ¿cuales son esas razones para el pluralismo? ¿cuáles son las razones que invitan a hablar de muchos mundos en lugar de uno solo? La respuesta de Goodman es ésta: lo que distingue algo como un nuevo mundo en lugar de como otra versión del mismo mundo no puede ser establecido de una vez por todas. A la distinción entre versiones de un mismo mundo, por un lado, y mundos distintos, por otro, le octurre lo mismo que a la distinción entre esquema y contenido. Tales distinciones son arbitrarias. $O$, en otras palabras, no tienen ninguma importancia ontologica destacable. Lo que haria de algo una versión sería su particular presentación, a través de cierto esquerna conceptual concreto, de un mismo contenido que se supone compartido por el resto de las versiones. Cada versión presentaría ese mismo contenido a través de versiones diferentes. Pero no hay normas inquebrantables que impidan considerar a un determinado rasgo como constitutivo de un contenido en lugar de como parte de un esquema, ni viceversa. El rechazo de este dogma del empirismo, la distinción entre esquema y contenido, así como de los dogmas de la distinción analítico/sintético y de la existencia de una basc empirica incontrovertible, está ciertamente en la base del pluralismo de Goodman. $Y$ las distinciones entre estilo y contenido, o entre convención y naturaleza, no serían sino otras formas camufladas de presentar los anteriores dogmas. No podemos destacar un mundo sobre los demás, haciendo de él la auténtica realidad de la cual el resto de los mundos sean versiones, porque podríamos hacer lo mismo con 
cualquiera de sus versiones. Hablar de versiones diferentes viene a ser, en definitiva, lo mismo que hablar de mundos distintos.

Todo lo anterior, sin embargo, no quiere decir que no haya mundos mejores y peores, más o menos correctos e incorrectos, ni tampoco que podamos construir cualquier mundo que queramos. Sólo quiere decir que ningún mundo correctamente construido tiene por sí mismo más realidad que los restantes.

Los capítulos 2, 3 y 4, titulados respectivamente "Sobre el estilo", «Sobre la cita" y " ¿Cuándo hay arte?", se ocupan de la creación de mundos artísticos. No obstante, muchas de las ideas que en ellos aparecen serían directamente aplicables a la construcción de cualquier mundo. Deben destacarse aqui dos cosas. En primer lugar, la insistencia de Goodman en la tesis de que, además de la denotación, hay otras importantes formas no lingüísticas de referirse a un mundo. Por ejemplo, la ejemplificación (de una idea, concepcion, etc.) y la expresión (de unos sentimientos, emociones, etc.). En segundo lugar, su tratamiento de la metáfora. La corrección de algo como las metáforas sólo es posible a través de falsedades literales. Esto tiene consecuencias importantes, pues si cosas como las metáforas, las generalizaciones, las formas no denotativas de referencia, etc., están siempre presentes en nuestra construcción de mundos, los criterios de correccion de nuestras construcciones deberán tener en cuenta más aspectos que la verdad. Estas ideas se desarrollan más extensamente en los capítulos 6 y 7 del libro bajo los títulos respectivos "La fabricación de los hechos" y "Sobre la correcta interpretación».

Como ya hemos indicado en varios lugares, Goodman no quiere ser sim- plemente un relativista. Con otras palabras, con sus mismas palabras (véase el Prólogo del libro), sólo sería un relativista radical bajo rigurosas restricciones. No todo vale. No podemos construir los mundos que queramos. Solo construimos mundos sobre mundos ya existentes. Además, si bien no podemos recurrir a la idea de que haya hechos objetivos que se encuentran o descubren mientras que las ficciones y versiones las construimos nosotros, hay construcciones mejores $\mathrm{y}$ pcores. La noción de correcciốn sigue vigente. Una noción de corrección que es mucho más amplia que la noción de verdad.

\section{Como ser pluralistas sin ser relativistas?}

A lo largo de todo el libro, a veces uno desearía encontrar alguna mayor aclaración respecto a la diferencia que debe haber entre considerar algo como correcto o real $y$, por otra parte, que ese algo sea correcto o real. Goodman tal vez contestara, como también contestaria Hilary Putnam desde su «realismo interno, ${ }^{3}$ que esa diferencia sólo puede hacerse dentro de cada mundo, que cada mundo tiene en sí mismo su propio ideal de corrección y realidad, que tal vez algún mundo particular consiga englobar a otros muchos extendiendo así sus propios criterios de corrección y realidad, pero que, incluso a pesar de esto último, querer señalar definitivamente, de una vez por todas, a un mundo particular como el mundo indiscutiblemente correcto $y$ real es absurdo.

Examinemos más de cerca este último punto. En su recensión al libro de Goodman que estamos comentando, Quine $^{9}$ intentaba poner un límite a su proliferación de mundos señalando los privilegios de la física. Recojamos un 
fragmento de la argumentacion de Quine:

Se tiene la impresion de que esta sccuencia de mundos o versiones se desploma en la absurdidad. ${ }^{10}$ Considero que la defensa que hace Goodman de ella consiste en afirmar que no hay ningún punto intermedio razonable en el cual ponerle un alto. Yo le pondria el alto después del primer paso: la teoría física. Concedo la posibilidad de teorias físicas alternativas sobre las cuales no podemos pronunciamos; pero el resto de su secuencia de mundos o versiones de mundo me parece sólo una metafóra más bien débil.

¿Por qué esta especial deferencia hacia la teoría fisica?, pregunta Goodman. Ésta es una buena pregunta y una parte de su mérito es que admite una buena respuesta. La respuesta no es que todo lo que vale la pena decir puede ser traducido al vocabulario técnico de la física; ni siquiera que toda buena ciencia puede traducirse a ese vocabulario. La respuesia es, en cambio: nada ocurre en el mundo, ni la vibración de un párpado, ni el aleteo de un pensomiento, sin una redistribución de estados microfisicos. Es normalmente inútil e insensato determinar exactamente cuáles estados microfísicos desaparecen y cuáles otros sobrevienen en cada evento, pero tiene que haber alguna barajadura en ese nivel; la fisica no puede afimar menos que eso. Si el fisico sospechara que hay atgun evento que no consiste en una redistribución de los estados elementales admitidos en su teoria física, buscaria tha foma de completar su teoria. En este sentido, llegar a cubrirlo todo es el negocio mismo de la fisica y sólo de la fisica [...] De ahí mi especial deferencia hacia la teoria física como versión del mundo y hacia el mundo físico como el mundo.

Confio en que habrá merecido la pena una cita tan extensa. El argumento de Quine se apoya en la idea de que cualquier variación o cambio en el mundo debe entrañar un cambio físico, una variación en los estados microfísicos del mundo. A veces se expresa esta idea diciendo que todo sobreviene a lo físico."

Como se desprende de parte del texto (primer subrayado), esa relación de sobreveniencia, tal como la entiende Quine, sería en sí misma un asunto exclusivamente ontológico. No solo no se requiere ninguna sinonimia entre las expresiones que describan algo sobreveniente $y$ aquellas expresiones que puedan llegar a describir la base microfísica de tal sobreveniencia. $\mathrm{Ni}$ siquiera se exige que las relaciones de sobreveniencia deban ser expresables en ningún lenguaje. Tales intuiciones resultan sugerentes; y sin embargo, no son incontestables. Pues, como Quine mismo afirma en las últimas frases del texto (segundo subrayado), aunque esa sobreveniencia sea en sí misma un asunto puramente ontológico, el interés que para nosotros pueda tener afirmar la sobreveniencia sobre lo físico de cualquier otro fenómeno y las razones para aceptar tal sobreveniencia sí presuponen un lenguaje y cierto límite ideal de la ciencia física. Y aquí empiezan los problemas. Porque ese límite ideal de la ciencia física es del mismo tipo que el que asimismo podría ser postulado, por ejemplo, para un sistema fenomenalista. Pero en este último caso, también podríamos decir que todo sobreviene sobre aquellos qualia del fenomenalismo a los que nos referíamos algo más amiba. ${ }^{12}$

La apelación a una sobreveniencia parece no favorecer a la física más que como una cuestión de hecho: los programas fisicalistas han tenido más desarrollos exitosos que los programas fenomenalistas (que los programas idealistas en general). Gran parte de nuestra historia como constructores de mundos se ha orientado por la idea de que merecía la pena señalar un mundo como el más correcto y real; a saber, el mundo de la física ( $o$, de una manera 
más vaga, el mundo material). Empeñados en esta obsesion hemos construido, destruido y vuelto a recomponer sistemas cosmologicos enteros.

Estoy de acuerdo en que querer señalar, definitivamente y de una vez por todas, a un mundo particular como el mundo indiscutiblemente correcto $y$ real es absurdo. Sin embargo, si cambiáramos el anterior «definitivamente y de una vez por todas» por un "mientras dure satisfactoriamente según nuestros criterios y conocimientos disponibles" (cosa sumamente razonable en otros contextos), tal obsesión dejaría de ser tan absurda.

De todas formas, ese "mientras dure satisfactoriamente según nuestros criterios y conocimientos disponibles" no puede tener, a su vez, una lectura demasiado restringida. Imaginemos (1) que restringimos el significado de ues verdadero" de manera que estipulemos que algo sea $S$-verdadero si y sólo si es verdadero para cierto sujeto o grupo cultural $S$. Imaginemos también (2) que, en un sentido parecido, lo que es verdad para $S$ no lo es para otro sujeto o grupo cultural $S^{\prime}$, por ejemplo, para nosotros. Llamemos $S^{\prime}$-verdadero a lo que, en este caso, es verdadero para nosotros. Si ahora nos preguntáramos (nosotros, es decir, $\left.S^{\prime}\right)$ qué cosas son verdaderas para $S$, la única respuesta posible sería que para $S$ es verdad aquello que según nuestros propios criterios es verdad para $S$. Sería verdadero para $S$ aquello que según nosotros es verdadero para $S$. Es decir, sería $S$-verdadero parte de aquello que es $S^{\prime}$ verdadero. Paralelamente, sería verdadero para nosotros aquello que scgún $S$ es verdadero para nosotros. Es decir, sería $S$-verdadero parte de lo que es $S$-verdadero. Pero, estos resultados contradicen muestra suposición (2). Fácilmente podríamos construir casos semejantes respecto de lo correcto, lo real, etc.

Las únicas formas de escapar de esta situación contradictoria parecen consistir en rechazar la anterior suposición (2), y las suposiciones análogas para lo correcto, lo real, etc., o bien en negar que tenga sentido preguntamos por lo que es verdadero (correcto, real, etc.) para otros $y$, con ello, por lo que puede hacer de ellos, justamente, personas como nosotros. ${ }^{13}$ Esta segunda opción vuelve a conducirnos al relativismo, la primera nos compromete con la convergencia última de lo que cada cual piense que es la verdad (lo correcto, la realidad, etc).

Esta última alternativa parece ser la adoptada por Putnam a fin de rescatar su internalismo del inmediato relativismo al que otras posiciones hasta cicrto punto similares se verian sujetas. ${ }^{14}$ También es una alternativa muy cercana al planteamiento de Goodman. Sin embargo, hay diferencias importantes. Mientras que para Putnam esa convergencia última es algo asf como un postulado de la razón, postulado que, en último término, se apoyaría en nuestra idea de bondad, para Goodman es algo sumamente frágil y contingente que también se debe construir.

El proyecto constructivo de Goodman, ese proyecto constructivo iniciado ya en The Structure of Appearence, se adopta hasta sus ultimas consecuencias. Se trata de un proyecto radicalmente opuesto tanto a los relativismos deconstruccionistas postmodernos como a las, a veces un poco ingenuas, vueltas a Kant. Su manera de ver las cosas tiene implicaciones importantes en todo cl debate actual en torno al pluralismo y al relativismo en ética y filosofía política. Si Goodman está en lo cierto, será más que nunca verdad aquello de que tenemos el mundo que nos merecemos. Claro que, si Goodman también está en lo cierto al negar cualquier preexistencia de lo posible, asimismo será verdad que el mejor de 
los mundos está siempre a nuestro alcance. Contemos con los materiales, con los mundos previos, con los que contemos.

Es muy difícil permanecer impasible al empuje de los argumentos e intuiciones de Goodman. Nelson Goodman nos ha construido un universo del que resulta muy dificil escapar. $Y$ resulta muy difícil escapar porque sus límites son indeterminables.

Tal vez sea éste, justamente, el principal motivo de desasosiego. Los ain- numerables mundos, creados de la nada mediante el uso de símbolosn de Cassirer y Goodman (Goodman comienza su libro con esta cita de Cassirer), pueden llegar a constituir un universo tan inhóspito como el más austcro universo fisicalista $\mathrm{y}$, también, pueden llegar a cuajar en un universo más desconcertante que el que jamás hayan llegado a imaginar los postmodernos. $Y$, sin embargo, no hay nada tan realista y seguro como aprender a confiar en nuestras propias fuerzas.

\section{REFERENCIAS}

Derrida, J. (1967a): Lecriture at le difference, Paris, Seuil.

- (1967b): La voix et le plénomene, Paris, PUF.

Feverabend, P. (1970): Agahist Method: Outine of an Anarchistic Theory of Knowledge, Minneápolis, University of Minnesola Press (Minnesota Studies in the Philosophy of Science, vol. IV). [Trad cast. en Barcelona, Ariel, 1974.]

Fovcault, M. (1966): Les mots et les choses, París, Gallimard. [Trad. cast. en Mexico, Siglo XXI, 1968.]

Goodman, N. (1972): Problems and Projects, Indianapolis, Hackett Publishing $\mathrm{Co}$.

- (1976): Languages of An, Indianápolis, Hackett Publishing Co. [Trad, cast, en Barcelona, Seix Barral, 1976.]

- (1977a): The Structure of Appearence, Dordrecht, Reidel Publishing $\mathrm{Co}$.

- (1977b): Fact, Fictiont, and Forecast, Indianapolis, Hackett Publishing Company, Inc.

- (1978): Ways of Worldmaking. Indianápolis, Hackett Publishing Compary, Inc.

- (1984): Of Mind and Other Matters, Cambridge, Harvard University Press.

Hempel, C. (1945): :Studies in the Logic of Confirmation", Mind, 54. [Recogido también en C. Hempel, Aspects of Scienific Explanation. Nueva York, The Free Press, 1970.]

Kim, J. (1984): Concepts of Superveniences, Philosophy and Phenomenological Research, XIV, 2.

- (1990): aSupervenience as a Philosophical Concepts, Metaphilosophy, 22, 1-2.
Mouluses, U. (1973): La estmetura del mundo sensible, Barcelona, Ariel.

Putnam, H. (1981): Reason, Truth, and History, Cambridge, Cambridge Univ, Press. [Trad. cast. en Madrid, Tecnos, 1989.]

- (1983): Reatisn and Renson, Cambridge, Cambridge Univ. Press.

- (1987): The Many Faces of Realism, La Salle, Open Court.

- (1990): Realism with a Human Face, Cambridge, Harvard University Press.

Quine, W. (1981): Theories and Things, Cambridge, Harvard University Press. [Trad. cast. en México D.F., Universidad Autonoma de México.]

RawLs, J. (1971): A Theory of Justice, Cambridge, Harvard University Press. [Trad cast. en MexiCO D.F., FCE, 1978.]

RonTy, R. (1979): Philosophy and the Mirror of Nature, Princeton. Princeton University Press. [Trad. cast. en Madrid, Cátedra, 1983.]

- (1982): Consecuences of Pragmatism. Minneapolis, University of Minnesota Press.

- (1985): "Solidarity or Objectivity", en J. Rajchman y C. West (eds.), Post-Analytic Philo. sophy, Nueva York. Columbia University Press.

Sosa, E. (1991): Knowledge in Perspective, Cambridge, Cambridge University Press.

Srich. S. (1990): The Fragmentation of Reason, Cambridge. MIT Press.

VV.AA. (1984): Southen Joumal of Philosophy, 22 [Suplemento monografico sobre sobreveniencia]. 


\title{
NOTAS
}

1. Véase Goodman (1977a) para la tercera edición de este libro. Su tesis doctoral llevaba por título *A Study of Qualitiesn.

2. Un análisis pomenorizado de los sistemas fenomenalistas de Mach, Camap, Russell y Goodman, así como una discusion critica del alcance y supuestos de todo el programa fenomenalista se encuentran en el libro de Moulines (1973).

3. Véase la tencera edición de este libro en Goodman (1977b).

4. El procedimiento de Goodman ha sido ampliamente utilizado dentro y fuera de la filosofia analítica. Tal vez, el caso más conocido sea la aplicacion que Rawls (1971) hace de cierto *quilibrio reflexivo en sentido amplio* a problemas de filosofia del derecho y filosofía política. La critica más mordaz a este tipo de estrategias la podemos encontrar en el capitulo 4 del reciente libro de Stich (1990). Una, a mi modo de ver convincente, respuesta a las criticas de Stich aparece en el capitulo 15 del también reciente libro de Sosa (1991).

5. Véase, por ejemplo, Hempel (1945).

6. Véase la segunda edición de este libro en Goodman (1976).

7. Problemática que se repite tambien en otras dos obras suyas que no hemos comentado, coodman (1972 y 1984).

8. Como inmediatas referencias bibliognficas respecto al srealismo interno de Hilary Putnam. destacańa Putnam (1981, 1983, 1987 y 1990).

9. Quine, "Goodman's "Ways of Worldmaking" ". New York keview (25 de noviembre de
1978). Los subrayados son míos. Este texto se encuentra reimpreso en Quine (1981). La cita pro. viene de la traducción castellana de este último libro. Un interesante intercambio de comentarios entre Quine, Goodman y otros aparecio ol $25 \mathrm{de}$ enero y el 17 de mayo de 1979 tambien en Now York Review.

10. Como se ve, las acusaciones recíprocas de absurdidad son sintoma indiscutible de que nos encontramos entre filosofos.

11. La actual discusion filosófica de este concepto de sobreveniencia o superveniencia (disponemos de estos dos terminos en castellano para traducir la palabra inglesa supervenience) comienza a resultar ya desbordante. Indjcaré sólo tres referencias bibliograficas: $\operatorname{Kim}(1984$ y 1990) y VV.AA. (1984).

12. Este problema es señalado, con gran acierto, por Putnam en su recensión del libro de Goodman que se recoge en Putnam (1983) bajo el título aReflections on Goodman's "Ways of Worldmaking" a. En esa misma recensión se analizan y rechazan diversas posibles réplicas fisica. listas al pluralismo de Goodman que, en buena medida, Putnam mismo comparte.

13. He adaptado aquí algunos argumentos de Putnam (1981) y Sosa (1991).

14. Posiciones como, por ejemplo, la que a veces adopta Rorty (1979, 1982 y 1985), o la que podemos también encontrar en autores como Feyerabend (1970), Foucault (1966) a Derrida (1967a y b).

\section{La caligrafía de la Historia}

\author{
M. TERESA LÓPEZ DE LA VIEJA \\ Universidad de Salamanca
}

Posiblemente uno de los problemas fundamentales del lenguaje filosófico consiste en un doble desarraigo. Por un lado, de la experiencia uvitals que haya puesto en marcha la reflexión filosófica, y que apenas si es perceptible ya en la escritura. Por otro lado, de la marcha propia de un discurso que se sostiene a sí mismo, estableciendo una cierta forma de coherencia en el engarce y justificacion de sus proposiciones. Sin embargo, la aparente neutralidad de ese discurso, y la «logica" de sus articulaciones, mantienen todavia formas de vinculación con el lenguaje natural. El olvido de su propia historia, al caer el lenguaje de la filosofía en el presente eterno de un tiempo 